\title{
The influence of Trichosanthin on the induction of IgE responses to ovalbumin under adjuvant-free condition
}

\author{
JI Yongyong, Cuinong Y ANG, Ming YEH \\ Shanghai Institute of Cell Biology, Chinese Academy of \\ Sciences, Shanghai 200031, China.
}

\begin{abstract}
Trichosanthin(TCS) is a potent allergen in mice. It can reproducibly induce specific $\operatorname{IgE}$ responses in C57BL /6J mice without the help of adjuvant alum. TCS can bring out the IgE responses to ovalbumin(OVA), while OVA itself could not induce IgE responses to it. However, TCS only works when OVA immunization is given one day after TCS immunization. Either time lag in OVA immunization, or immunization of both antigens at the same time, or OVA immunization given first, all has no effect on the induction of IgE responses to OVA. Through analysis of the antibody specificity of hybridoma clones, it indicated that specific antibodies to TCS or OVA were secreted by independent B cell clones. The IgE antibodies showed no polyreactivity to different antigens.
\end{abstract}

Key words: Trichosanthin, ovalbumin, allergen, $\operatorname{IgE}$ response.

\section{INTRODUCTION}

Trichosanthin (TCS) is an effective protein component of a Chinese herb medicine, which, has long been used in history for abortion induction[1]. Recently it has been found that TCS has anti-HIV effects[2], which is arousing much interest in it. Its amino acid sequence, DNA sequence, and 3D structure have all been worked out[3, 4]. In clinical application it occasionally induce anaphylaxis in certain patients[5]. Previously we had done a number of studies on the TCS specific IgE responses in mice immunized with TCS and adjuvant alum[6]. Since using the protein alone can induce anti-TCS IgE responses in human body, we intend now to study the TCS-IgE

1. The work is specifically dedicated to Prof. Zhen YAO for his 80-yeaxs birthday 
TCSon the induction of $\operatorname{IgE}$

responses without the influence of adjuvant in mice. That would be possibly more closer to the status happened in human body. Furthermore cross-reactivity of $\operatorname{IgE}$ antibodies in sera with different allergens in some of common allergenic determinants on different allergens is suggested to be the reason. However that it may be due to certain structural characteristics in antigen binding site of IgE molecules was also suggested[8]. And the possibility of allergens may influence different lymphocyte clones through mediators or cells that involved in the induction of $\operatorname{IgE}$ antibody responses within microenvironment could not be excluded also. In this paper we intend to establish a reproducible TCS-specific IgE antibody responses without the use of adjuvant to investigate its allergenic potential, and try to study the cross reactivity of allergen at cell clone level.

\section{MATERIALS AND METHODS}

\section{Animals}

1. Mice: C57BL/6J mice, female and 12 weeks of age

2. Rats: Wistar rats, female with $200 \mathrm{~g}$ body weight.

\section{Reagents}

\section{Antigens}

1) TCS: Crystalized TCS in Trichosanthin Injection Solution (TCS-J), produced by Jin-San Pharmaceutical Factory, $1.2 \mathrm{mg} / \mathrm{ml}$, was used as immunogen. Refined TCS (TCS-W), produced by Wu-Han Biological Products Research Institute, $2 \mathrm{mg}$ /ampule was used in the passive cutaneous anaphylaxis(PCA) test as a challenger and in ELISA as a coating antigen.

TCS-J has now been commonly used in clinics for a number of years. Considering TCS-J induced immune responses would be more closer to clinical status, we used it as the immunogen. However we used TCS-W as a challenger to detect the formed TCS-J-specific IgE reponses in mice. The reason is that the source of TCS-W is available and abundant in our hands. And we have intentionally run parallel experiments to compare the PCA results following TCS-J or TCS-W challenge. The results showed no differences (unpublished data).

2) Ovalbumin (OVA), produced by Shanghai Dong-Feng Factory for Reagents.

2. Evan's Blue, purchased from Shanghai Chemical Reagent Company. It was dissolved in phosphate buffered saline (PBS) and was adjusted to $1 \%$ concentration ready for use.

\section{Immunization}

The TCS-J or OVA were diluted or dissolved in PBS respectively and was each adjusted to 10 $\mu \mathrm{g} / \mathrm{ml}$ and $20 \mu \mathrm{g} / \mathrm{ml}$. Each mouse was intraperitoneally injected with $0.5 \mathrm{ml}$ antigen solution. The protocol of immunization was referred in below.

\section{Assays of the antibody specificity and titer}

1. ELISA: Indirect ELISA was used to detect the specific antibody in antiserum or in hybridoma culture supernatant. The procedure were briefly as follow.

TCS-W or OVA were used as the coating antigens. 1:50 diluted antiserum or hybridoma culture supernatants were used as the first antibody . Peroxidase labelled goat anti-mouse Ig (anti-MIg) or goat anti-mouse IgE (anti-MIgE) were used as the second antibody. Tetramethyl benezidine (TMB) and hydrogen peroxide were used as substrate.

2. PCA: The assay was used to detect the antigen-specific IgE antibody in antiserum or hybridoma culture supernatant. According to Ovary[9], the assay was carried out briefly as follows. 
On the shaved back of rats, $0.1 \mathrm{ml}$ antiserum diluted to $1: 10$ with PBS or $0.1 \mathrm{ml}$ of hybridoma culture supernatant were intradermally injected for priming. On the next day $2 \mathrm{mg}$ TCS-W or 3 mg OVA dissolved in $1 \%$ Evan's blue were intravenously injected into the tail vein of the rat. After 30 minutes blue patches appeared at the primed site were accounted as positive reaction, i.e. the presence of antigen-specific IgE in the test samples.

\section{Construction of hybridomas}

The primed lymphocytes were from mesenteric lymph node of mice immunized according to immunization protocol for Group B (see below). Five days before fusion the mice from Group B were boosted again. The tumor fusion partner was either SP2 /0 or NS-1 myeloma ceils. The fusion was carried out according to the routine method, including selection by HAT medium and screening.

\section{RESULTS}

Does minute amount of TCS alone without adjuvant could induce anti-

\section{TCS IgE responses?}

The ELISA results of TCS specific serum titer from mice immunized ip with $5 \mu \mathrm{g}$ TCS-J at 2-week interval are shown in Tab 1. It shows that all mice after two immunizations produced anti-TCS antibodies. And the antisera also contained anti-TCS IgE antibodies since PCA results indicated positive reaction.

Tab 1. ELISA and PCA results of antisera* from C57BL/6J mice immunized with TCS-J

\begin{tabular}{|c|c|c|c|c|c|c|c|c|c|}
\hline \multirow{2}{*}{$\begin{array}{c}\text { Times of } \\
\text { immunization }\end{array}$} & \multirow{2}{*}{$\begin{array}{c}\text { Days after } \\
\text { immunization }\end{array}$} & \multicolumn{4}{|c|}{ ELISA（ig） $(\mathrm{OD} \pm \mathrm{SD})$} & \multicolumn{4}{|c|}{ PCA(IgE) } \\
\hline & & $1^{* *}$ & 2 & 3 & Control & 1 & 2 & 3 & Control \\
\hline \multirow[t]{4}{*}{1} & 7 & 0.12 & 0.12 & 0.13 & 0.26 & & & & \\
\hline & & \pm 0.01 & \pm 0.01 & \pm 0.01 & \pm 0.15 & & & & \\
\hline & 14 & 0.16 & 0.21 & 0.63 & & - & - & + & - \\
\hline & & \pm 0.01 & \pm 0.09 & \pm 0.02 & & & & & \\
\hline \multirow[t]{4}{*}{2} & 7 & 0.75 & 0.75 & 0.71 & & + & + & + & \\
\hline & & \pm 0.01 & \pm 0.04 & \pm 0.01 & & & & & \\
\hline & 14 & 0.84 & 0.85 & 0.77 & & + & + & + & \\
\hline & & \pm 0.01 & \pm 0.01 & \pm 0.05 & & & & & \\
\hline \multirow[t]{4}{*}{3} & 7 & 0.97 & 0.98 & 0.99 & & + & + & + & \\
\hline & & \pm 0.03 & \pm 0.01 & \pm 0.03 & & & & & \\
\hline & 14 & 1.00 & 1.03 & 1.00 & & + & + & + & \\
\hline & & \pm 0.02 & \pm 0.06 & \pm 0.02 & & & & & \\
\hline
\end{tabular}

* Antisera were diluted to 1:50 or 1:10 with PBS used as test samples in ELISA and PCA respectively.

** The numbers of mice.

\section{Does TCS effect the induction of OVA-specific IgE responses?}

Through immunization of different combination of TCS-J and OVA, the influence of TCS on the induction of OVA-specific IgE responses in C57BL/6J mice could be 
TCSon the induction of $\operatorname{IgE}$

observed. The protocol for the combined immunization of TCS-J and OVA is shown in Tab 2.

Tab 3 shows the experimental results. Results from Group $\mathrm{F}$ show that OVA alone could not induce IgE responses in mice. However, under the influence of TCS, it could induce OVA-specific IgE responses (Group B). But TCS effects only when it was used one day before OVA immunization. In case of TCS used five days before, it showed no promotion effects on the induction of OVA-specific IgE responses(Group A). On the other hand, either TCS and OVA given at the same time (Group C) or OVA given first and then immunization with TCS (Group D), none could induce OVA-specific IgE responses, although the anti-TCS IgE responses were all induced in these groups. These results have been repeatedly reproduced in separate experiments.

Tab 2. Protocol of combined immunization of TCS-J and OVA

\begin{tabular}{cccccccc}
\hline \multirow{2}{*}{$\begin{array}{c}\text { Time } \\
\text { (Day) }\end{array}$} & A & B & C & D & E & F & G \\
\cline { 2 - 8 } & TCS* & - & - & - & - & - & - \\
4 & - & TCS & - & - & - & - & - \\
5 & OVA & OVA & OVA+TCC & OVA & TCS & OVA & - \\
6 & - & - & - & TCS & - & - & - \\
19 & TCS & - & - & - & - & - & - \\
21 & - & TCS & - & - & - & - & - \\
22 & OVA & OVA & OVA+TCS & OVA & TCS & OVA & - \\
23 & - & - & - & TCS & - & - & - \\
35 & & \multicolumn{7}{c}{ Bleeding } & & & \\
\hline
\end{tabular}

* The antigens used in immunozation.

Tab 3. PCA results of antisera** from C57BL/6J mice immunized with TCS-J or OVA or both

\begin{tabular}{cccccccc}
\hline & \multicolumn{1}{c}{ Groups of mice* } \\
\cline { 2 - 7 } Challenger & & & & & & \\
(Antigen & A & B & C & D & E & F & G \\
TCS & + & + & + & + & + & - & - \\
OVA) & - & + & - & - & - & - & - \\
\hline
\end{tabular}

* The grouping of mice was shown on Tab 2 .

** The antisera were diluted to 1:10 with PBS and used for intradermal sensitization of rats.

Anti-OVA IgE and anti-TCS IgE are from separate Nones or from one clone?

The hybridomas constructed by the fusion of mesenteric lymph node cells from Group B mice and myeloma cells were tested for their antibody specificities against TCS and OVA. Tab 4 shows the results of specific antibody titer of antiserum from 
lymphocyte donor. It can be seen that both antibodies against TCS and antibodies against OVA are present, and both contains TCS- and OVA-specific IgE, since the PCA tests are both positive.

The specificities of the antibodies secreted by hybridomas in separate wells tested in two experiments are listed in Tab 5. In Experiment 1, although there are some wells that are both TCS and OVA positive in Ig-ELISA, not a single well shows both positive reaction to TCS and OVA in PCA test. It means that there is not presence of both anti-TCS and anti-OVA IgE activities in a same well. In fact only five OVA-specific IgE wells were detected. Since the fusion rate is quite low , $18.4 \%$, it can assume that each well contains a single clone, and the anti-TCS or anti-OVA $\mathrm{IgE}$ antibodies are secreted by individual clones. There is no indication of the cross reactivity or polyreactivity at the antibody molecule level. Experiment 2 further provides evidence for the assumption. This time there only appears two wells that are both TCS and OVA positive in the IgE-ELISA. And the majority of the wells are either TCS-IgE positive (72 wells) or OVA-IgE positive ( 75 wells). After further cloning of the cells in double positive wells, antibody secretion in one well was lost. All the wells growing out cells were negative. The other one turned to single positive. The cloning rate is $19 \%$. All the wells only showed positive reaction to TCS in IgE-ELISA without simultaneous positive reaction to OVA.

Tab 4. Antibody assay of the antisera from group $B^{*}$ mice immunized with TCS-J and OVA

\begin{tabular}{lcc}
\hline & \multicolumn{2}{c}{ Antigen } \\
\cline { 2 - 3 } Assay & TCS & OVA \\
ELISA(Ig) & $1: 6400-12800 * *$ & $1: 3200$ \\
PCA(IgE) & + & + \\
\hline * See Tab 2. \\
** Number of dilutions when OD value \\
\multicolumn{2}{c}{ was over 0.1.}
\end{tabular}

\section{DISCUSSION}

It has well been known that IgE mediates immediate hypersensitivity, an allergic response. When allergens cross-link the allergen-specific IgE receptors on cell surface of mast cells or basophils, it will activate these cells, leading to the release of allergic mediators, thus bringing about the allergic responses. Therefore induction of $\operatorname{IgE}$ antibody responses is the characteristics of allergen. In the present study, it indicates that minute amount of TCS can reproducibly induce specific IgE responses in mice. TCS is a potent allergen.

As a potent allergen, what about the allergic potential of TCS? For this, we studied the effects of TCS on the induction of IgE responses to other antigen, which by itself is not an allergen, but when it is used to immunize under the influence of 


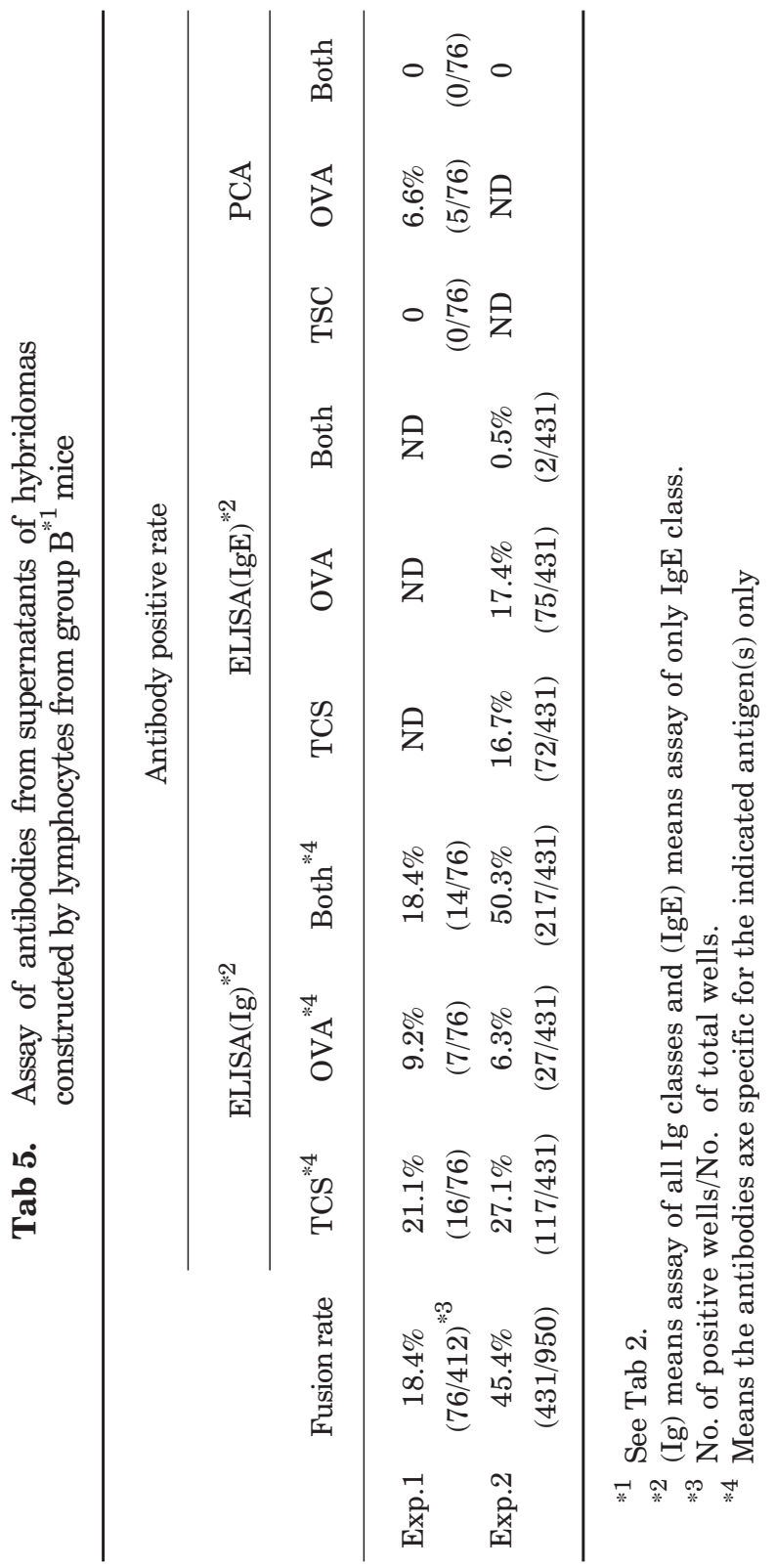


TCS, the consequence is different. The present study indicates TCS could bring out IgE responses to OVA, which normally is not an allergen. In our experiments also showed that immunization with OVA alone could bring about OVA-specific antibody responses (unpublished data) but not IgE responses. However when OVA was used in combination with TCS, OVA-specific IgE responses were aroused. Thus it indicates that TCS enables other unrelated antigen-specific B lymphocyte clones to secrete specific IgE antibodies, while they normally do not switch to secrete IgE antibody. The mechanism seems not to be due to the presence of common antigenic determinants on TCS and OVA, since they are structurally quite different. And it seems not to be due to polyreactivity or multispecificity of the antigen binding sites on the IgE antibody molecules, since through the detection of the antibody specificity at clonal level (hybridoma clones), the secreted antibodies of hybridoma cells in each well were all antigen specific, i.e. they only reacted with one antigen, either TCS or OVA. Double positive reaction of antibodies secreted by hybridoma clones have not been found.

In the process of immune responses, some aspects are antigen-specific, but some are non-specific. However the non-specific ones also play very important roles in immune responses. As we all know that interleukin-4 plays a very important role in $\operatorname{IgE}$ switch[10,11]. Some other cytokines and cell surface molecules are shown to be also involved in the regulation of $\mathrm{IgE}$ responses[12]. After the allergic state created by TCS, when another antigen circulates to the "allergic" micro-environment, the antigen-specific lymphocyte cell clones not only received signals from specific antigen but may possibly also receive some signals already present in the micro-environment, which just critical and appropriate for the induction of $\operatorname{IgE}$ switch in B cells at the particular stage. In this case OVA-specific clones may receive the appropriate nonspecific signals, IL-4 etc presumably, resulted from TCS stimulation. They may drive the OVA-specific B cell clones to switch to IgE secretion. The present research lend some support to this possibility, since the condition leading to $\operatorname{IgE}$ switch in $B$ cells is very critical. Only when mice were immunized with OVA one day after TCS was given, the OVA-specific IgE responses can be induced. In this sense it may represent a bystander effect. Furthermore it has been reported that ricin can enhance the IgE responses to an allergen, bee venom phosphilipase A2 (PLA2)[13]. They showed it was due to suppression of CD8 $\mathrm{T}$ cells by ricin. Ricin has not been reported to be an allergen and to induce $\operatorname{IgE}$ response. It is a toxic protein, while TCS is also a toxic protein, and is reported to be structurally related to ricin A chain[14]. Whether TCS also through the suppression of CD8 T cells to bring out the induction of $\mathrm{IgE}$ responses is interesting to study. However, there are also reports that showed TCS could activate CD8 T cells to mediate TCS immune suppression[15].

Anyway, whatever the true mechanism to be, the present paper demonstrates that a potent allergen can bring out the IgE responses to another unrelated antigen, even the antigen originally is not an allergen itself. This may afford an explanation 


\section{TCSon the induction of IgE}

for the cross-reactivity of IgE antibodies in sera with different allergens, which are frequently reported in allergic patients. The "cross-immunization" of allergens may lead to the persistent allergic status. Further study of the cellular and molecular mechanism of the "cross-immunization"potential oi' allergens may provide clues to the new strategy for the prevention and treatment of allergic diseases, which almost affect $10-15 \%$ of the people.

\section{ACKNOWLEDGMENT}

The authors thank Ms. Lin Guomei and Mr. Xie Zhigang for their technical assistance.

\section{REFERENCES}

[1] 2nd lab. of Shanghai Institute of Experimental Biology. Investigation of principle of trichosanthin induced abortion. Scientia Sinica 1976; 2:200.

[2] McGrath MS et al. An inhibitor of human immunodeficiency virus replication in acutely and chronically infected cells of lymphocyte and mononuclear phagocyte lineage. Proc Natl Acad Sci USA 1989; 86:2844.

[3] Collins EJ et al. Primary amino acid sequence of trichosanthin and molecular models for abrin A-chain and -trichosanthin. J Biol Chem 1990; 265:8665.

[4] Gao B et al. The structural determination its rectification of trichosanthin at $1.73 \mathrm{~A}$ resolving power Science in China (series B) 1993; 23:273.

[5] Wang SF et al. The study and application of trichosanthin. 1979: Science Publisher.

[6] $\mathrm{Gu} \mathrm{H}$ et al. Preparation, isolation and characterization of mouse IgE monoclonal antibodies against trichosanthin protein. Acta Biol sinica 1986; 19:109.

[7] Baldo BA and Donovan GR. The structural basis of allergenicity: recombinant DNA-based strategies for the study of allergens. Allergy 1988; $\mathbf{4 3 : 8 1 .}$

[8] Kofler $\mathrm{H}$ et al. Mechanism of allergic cross-reaction -III.cDNA cloning and variable-region sequence analysis of two IgE antibodies specific for trinitrophenyl. Mol Immunol 1992; 29:161.

[9] Ovary Z. Passive cutaneous anaphylaxis in the mouse. J Immunol 1985; 81:355.

[10] Finkelman FD et al. IL-4 is required to generate and sustain in vivo IgE responses. J Immunol 1988; 141:2335.

[11] Finkelman FD et al. Lymphokine control of in vivo immunoglobulin isotype selection. Annu Rev Immunol 1990; 8:303.

[12] Ishizaka K. Regulation of immunoglobulin E biosynthesis. Adv Immunol 1989; 47:1.

[13] Diaz-Sanchez D et al. Elimination of IgE regulatory rat CD8 T cells in vivo differentially modulates interleukin- 4 and interferon-r but interleukin-2 production by splenic T cells. Immunology 1993; 78:513.

[14] Zhang XJ and Wang JH. Homology of trichosanthin and ricin A chain. Nature 1986; 321:477.

[15] Cho KY et al. Human immune suppression is inducible by trichosanthin via CD8 cell-mediated pathway. Cell Res 1994; 17.

Received 6-3-1995. Revised 5-5-1995. Accepted 13-5-1995. 